\title{
An Empirical Research on the Formation Mechanism of Overwork of Chinese Knowledge Workers
}

\author{
Wang Dan ${ }^{1}$ \\ ${ }^{1}$ State Grid Energy Research Institute, Beijing, China, 100052
}

\begin{abstract}
With reference to relevant theories of economics, management science, and psychology, this research classifies the influences that lead to Chinese knowledge workers' overwork into three categories: antecedent variables, determining variables and moderating variables, and constructs a conceptual model concerning its formation mechanism, which, taking off from Chinese knowledge workers' group features and individual features, makes an analysis of the correlations between these influences and their mechanisms of action. Empirical study on the knowledge workers in the CBD district of Beijing has partly verified this conceptual model.
\end{abstract}

Keywords: Knowledge Workers; Overwork; Death from overwork; Formation Mechanism

\section{Introduction}

Knowledge workers are workers who are occupied in production, creation, extension and application of knowledge, etc, and bring knowledge capital gain for their employers, such as business organizations, government agencies, and public institutions, and who, on the employers' knowledge value chain, undertake such non-structural tasks as knowledge acquisition and innovation, as well as its sharing and application.On the one hand, they are equipped with relevant knowledge and skills and directly engaged in knowledge-intensive work. On the other hand, they have knowledge acquisition and innovation, as well as its sharing and application as the main content of their jobs with a view to the organization's knowledge capital appreciation.

In recent years, death from overwork, a modern disease that originated in developed countries, has already settled itself in China which is undergoing modernization, industrialization, and informatization, with knowledge worker being highly vulnerable to its presence. According to a research conducted in November 2009 among the workers in enterprises and public institutions of Beijing,72.73\% of those interviewed suffer liability of accumulation of fatigue and are in need of having their working conditions improved, among these people, $27.64 \&$ being in "Danger zone" of overwork, $11.52 \&$ in "High-risk"zone. "Health Conditions of the white-collar workers in the Urban Areas of China"issued on July 6, 2009 showed : $76 \%$ of the urban white-collar workers in Mainland China are in the state of subhealth, nearly $60 \%$ in the state of overwork. Overwork, instead of moderate-work has already become the 
norm in the everyday work life of knowledge workers.

\section{The influence factors of overwork on the part of knowledge workers}

The problem of overwork on the part of knowledge workers is not without foundation. There must be a variety of factors leading to its occurrence and development. The consequence of the combined action of these various factors is the development and the gradual deterioration of the overwork status of knowledge workers.

\subsection{Antecedent variables}

The antecedent variables are the underlying causes of knowledge workers' excessive labor. They are of common significance to the whole labor force, including knowledge workers. When the underlying factors in line with the group characteristics of knowledge workers emerge, further specification will be given.

\section{- Cultural Dimensions in the Society}

Values based on patriotism, collectivism and socialism still have an important guiding role to play in contemporary China. They are in alignment with the objective requirements and the actual operation of the socialist market economy and serve as the realistic basis of the adjustment of a variety of value conflicts during the transitional period of the society. (Chen Xiaoying, 2009).

As a result, people voluntarily and spontaneously work intensively and excessively for long periods without a timeout, without suspecting that overwork may jeopardize themselves, their employers, the country, and the long-term, sustainable and harmonious development of the whole society, regarding it, instead, as a contribution to the country, the society and the economic development, compatible with the collectivism. This kind of unconscious overwork throughout the whole society will, in turn, exert its negative influence on the workforce that is in or will be in the labor market, have the whole workforce continue to pay the excess labor in the collective atmosphere.

\section{- Economical Dimensions in the Society}

At the present stage, the objective state conditions of low productivity, backward economy and culture, directly determine the economic and social development must be regarded as the first order of business in our country, which inevitably leads to a blind pursuit of a high growth rate of GDP on the part of the local governments, of a faster primitive accumulation of capital and interest appreciation on the part of the employers, and of a higher level of material comforts on the part of the workers, which again inspires the workers to overwork.

At the same time, during the period of economic transformation, modernization and urbanization is speeding up, and the various aspects of social life are undergoing an unprecedented shock, change and adjustment. The imbalance of the development of social economy becomes more significant. All kinds of pressure also increasingly permeate every level of our workers. All this promotes the occurrence and development of excessive labor in our country, and endows it with a homology, in terms of the social and economic driving force, with the problems of excessive labor undergone by the developed countries or regions such as Japan, Europe and the United States.

On the other hand, with the deep development of the globalization of the world economy, China has integrated into the global economic order, and become one of the key links in the global 
production and consumption. The consumption level has become one of the important measures of the value of an individual. In order to seek a more glamorous life, the workers cannot but prolong the working hours or increase the workload, to obtain relatively high human capital returns, i.e., labor remuneration, which means he falls into the state of excessive labor.

\section{- Social Institution as a Dimension}

This mainly lies in the unsoundness of relevant laws and regulations.

First of all, labor laws fail to implement the safeguard measures for the protection of workers'right of rest.Labor inspection departments are inert in their intervention in the case of employers compelling employees to work overtime. Some scholars think that the punishment given in labor laws and regulations about the employers' violation of the laborer's right of rest is too light ( $\mathrm{Lu} \mathrm{Lu}, 2003$ ).

Secondly, with the existence of legal loopholes, China's labor law system is not clear on the issue of "death from overwork". When death occurs due to overwork, the employers' responsibility for it and their infringement of the worker's right of rest are issues of controversy in the legal world. (Wang Lixia, 2008). In this case, the employers are likely to impose excessively long and harsh work hours on the workers in order to maximize their profits, at a relatively low management and legal price.

Finally, the poor performance in the promotion and popularization of the labor laws and regulations constitutes one of the reasons causing excessive labor.

\subsection{Decisive Variables}

Decisive variables play a decisive role in the occurrence, evolution and development of overwork.

\section{- Individual traits}

Individual traits mainly include individual choice, motivation, emotion, the level of effort, responsibility, mode of work, store of knowledge, learning ability, cognitive mode and skills, sense of selfefficacy, health conditions, personal knowledge management, team cooperation ability, ability to handle stress. On the premise of the influences of social culture, economy and institutions, knowledge workers arouse or curb their motives of overwork, in accordance with the characteristics of the individual, and overwork behaviors come into existence with a series of consequences.

Here, "conflicts among the three 'I's" is a breakthrough in the research on the individual factors affecting the issue of excessive labor of the knowledge workers. The three 'I's refer to the actual ME (self), work-involved ME (I as an actor or actress) and $\mathrm{ME}$ in the eyes of other people (ME relative to others). When the three 'I's are coordinated, the cost of effort and energy on the part of the workers in work is at a low level, the result of which is, in terms of their performance in work, they are more likely to maintain excellent performance, high productivity, and a stable and positive emotion; otherwise, they are more prone to fatigue and emotional frustration in work, and ultimately have their working efficiency and performance negatively affected. Further, in order that "I as an actor or actress" achieve the requirement of the" self-I" in terms of style, the knowledge workers may need to pay more time and energy in work than when the "self-I" and "I as an actor or actress" work in coordination, which results in excessive labor both in terms of time and labor intensity.

\section{- Management}

Management mainly contains the employers' management philosophy, incentive mechanism, leaders' behaviors and enterprise culture etc. Whether these factors are consistent with the value orientation and the group characteristics of knowledge workers will directly 
affects knowledge workers' willingness and the degree of the willingness to work, resulting in two entirely different situations:

one is that the knowledge workers' job involvement is reduced, which manifests as go-slow, reduction of the time, efficiency and quality of knowledge work; the other, knowledge workers's job involvement is enhanced, which shows up as their voluntary, spontaneous or conscious extension of working hours, their initiative to take more arduous tasks, increasing the intensity of labor. It is thus clear that management, given the group characteristics and the individual characteristics of knowledge workers, has an influence on the knowledge workers' motives of overwork, which give birth to the excessive labor behaviors.

Many employers cannot formulate reasonable working hours and workloads, and ignore the creation of a good working environment, enforce a bad management in the work process. Their human resources departments carry their main work according to the traditional personnel management method, and fail to strike up effective communications with knowledge workers. Also, there exists such practice as to regard working hours as a requirement of promotion or salary increase, so that knowledge workers lack work autonomy, and can not solve timely the difficulties in their work or in their life. All these factors result in the fact that workers are passive recipients of the enterprise's labor system, whose unreasonable factors often become the decisive variables of excessive labor on the part of the knowledge workers.

\subsection{Moderating Variables}

Moderating variables refers to the variables which do not play a decisive role in the emergence, evolution and development of knowledge workers' excessive labor, but which exert their influence still.
- Group characteristics of knowledge workers

A clear understanding of the group characteristics of knowledge workers can help to understand why knowledge workers continue to work overtime under great labor intensity when they feel tired or suffer mental and physical disorders. In general, knowledge workers' group characteristics proposed by the academic world mainly include relatively high work autonomy; high levels of education; more loyal to the occupation than to the organization; higher creativity; good at invisible labor; short renewal cycle of knowledge and skills. As can be seen, workers of this kind who seek to realize themselves, think highly of working autonomy, and need to constantly update their knowledge and skills are achievement-motivated on the one hand; on the other hand, they need work hard, even excessively hard in order to achieve self realization and meet the requirements of creativity embodied in work .

\section{Work task as influences}

These influences mainly include the complexity and explicitness of the work task and the matching between the task and knowledge workers' ability, which link the requirements of competency in the knowledge workers with the workers' motivation, emotion and behavior. If the task is too difficult or if there is a mismatch between the task and the knowledge workers' capability, then the workers' emotion and motivation will be affected, and then the knowledge workers' working hours and work intensity will be affected, and accordingly excessive labor occurs.

\section{The formation mechanism of overwork on the part of knowledge workers}

According to the psychological theory of achievement motivation, achievement 
motivation has a certain intrinsic logic connection with management, social environment factors, work task and therefore, account being taken of such group characteristics of knowledge workers as strong autonomy, seeking self realization, the analysis of the forming mechanism of excessive labor of knowledge worker is conducted as below:

Overall, the antecedent factors such as social culture, economy and institutions, working as a kind of environmental factors, exercise an invisible, formative influence on knowledge workers' motivation; individual characteristics have more or less a decisive role to play in bringing about knowledge workers' motivation, intention and behavior of overwork; management factors directly affect knowledge workers' motivation and behavior of over work, indirectly shape their willingness to overwork. Work task as a factor exerts its influence directly on individual factors which in turn affect the workers' over labor behavior.

\section{Empirical Study}

From September to November in 2010, by using the foreign mature "Copenhagen scale" and Karasek's "Load-control Model" and its derivative Chinese version " Job content questionnaire", a convenient sampling survey was conducted among knowledge workers in the CBD area of Beijing city mainly on the relationship between knowledge workers' work characteristics and excessive labor. A total of 300 questionnaires were released, and 228 collected, among which 194 were valid, effective rate being $85 \%$. Of all the respondents, men accounted for $41.8 \%$, and women accounted for $58.2 \%$. $57.7 \%$ were concentrated in the 26-40 age range, $54.6 \%$ with a bachelor's degree, covering 11 categories of occupation like scientific research, law, accounting / auditing, HR, physicians and others.

According to the reliability test standard established by Numnally (1975), Ehurehill and Peter (1984), if the Cronbach's Alpha coefficient is higher than 0.8 , then a relatively high internal consistency of the questionnaire is assumed; if the coefficient is between $0.7-0.8$, then the reliability has an acceptable value; if the coefficient is less than 0.7 , the scale should be revised. In this study, the Cronbach's Alpha coefficient test results of the questionnaires of this study are shown in table 1. Short form health scale, personal fatigue and service object's fatigue questionnaire had good reliability, and the internal consistency of other scales can be accepted.

Chart 1 The reliability analysis of the questionnaire

\begin{tabular}{|c|c|}
\hline $\begin{array}{l}\text { Test sub } \\
\text { questionnaire }\end{array}$ & $\begin{array}{l}\text { Cronbach's Alpha } \\
\text { coefficient }\end{array}$ \\
\hline $\begin{array}{c}\text { Mental load of } \\
\text { the work }\end{array}$ & 0.724 \\
\hline Job control & 0.744 \\
\hline Job insecurity & 0.747 \\
\hline $\begin{array}{c}\text { Short form } \\
\text { health scale }\end{array}$ & 0.805 \\
\hline $\begin{array}{c}\text { Individual } \\
\text { fatigue }\end{array}$ & 0.873 \\
\hline Work fatigue & 0.793 \\
\hline $\begin{array}{c}\text { Fatigue of } \\
\text { service object }\end{array}$ & 0.857 \\
\hline Work fatigue & 0.782 \\
\hline
\end{tabular}

Through relevant analysis, the study shows:

(1) There is small positive correlation between the load and pressure of work and the four fatigue scales.

(2) Work control factors do not generate individual fatigue and work fatigue, but they can produce fatigue of 
service object and work fatigue, which is consistent with the proposed formation mechanism. Job control factors include "During work, I need to learn new things", "I do a lot of repetitive work", "During work, I must be innovative", "During work, I can make my own decisions on a lot of things", "My work requires a high degree of technology". These work factors are rather challenging for the knowledge workers.

(3) Job insecurity factors will not induce personal fatigue and work fatigue, but it will produce fatigue of service object and work fatigue.

(4) There is a strong positive correlation between the subjective psychological distress and individual fatigue. It takes on a small positive correlation with the other three fatigue scales.

\section{Review and Prospect of the Research}

Overall, personal factors and management have a more or less direct effect on the motives, willingness and behavior of excessive labor of knowledge workers. Work task, social culture, economy, and institution, as underlying causes, constitute environmental factors, which serve as an incentive or a restraint on the knowledge workers, so that give certain over work motivation and willingness, they are more prone than other workers to pay excessive labor of super intensity and overtime, resulting in a variety of pathological or psychological manifestations of excessive fatigue. The results of the empirical study conducted among the knowledge workers in CBD area of Beijing partly verifies the theoretical model presented in this paper.

In the present era when the economy and the society of our country are still in a transitional stage of transformation, if the problems excessive labor of the knowledge workers are left to run their own course without any restraint, it is likely that a series of irreversible problems will evolve that run counter to the scientific outlook on development and the values and the attitude toward labor of a harmonious society, which in turn will affect the long-term, sustainable and healthy development of China's social economy.

Therefore, in further research on labor issues of the knowledge workers, on the one hand, an in-depth inquiry should be carried out, on the strength of a large number of empirical data, into the degree of influence and their operating laws of various factors affecting over labor of knowledge workers; on the other hand, a study can be conducted, with decisive variables and moderating variables as a point of departure, on the interference and prevention of knowledge workers's overwork, to discover theoretical support for the remission and solution of overwork, and promote the policy establishment or national legislation.

\section{References}

[1] Drucker P F. Knowledge worker Productivity: The Biggest Challenge [J]. California Management Review , 1999, 41 (2):79-94.

[2] Horibe F. Managing Knowledge workers $[\mathrm{M}]$. Translated by Zheng Xiaoming. Beijing: China Machine Press, 2000: 5-15.

[3] Liao Jianqiao, Wen Peng. A Research on the Definition, characteristics, and classification of Knowledge Workers.[J]Journal of Management.2009,1(2):277-283.

[4] Wang Dan, Yang Heqing. A Survey on the Situation of Overwork in the Enterprise and Public Institutions in Beijing. [J] Development of Human Resources of China.2010(9):38-40. 
[5] White book on the Health Conditions of White-collar workers in China [OL].http://www.yylm.org/contents/1 007/18830.html. 2009-12-11.

[6] Wang Dan. An Assessment of Chinese Workers' Overwork Based on an Empirical Study[J]. Economic Survey.2011(2):86-90.

[7] Zhang Chunyu, Zhang JInfu, Zhang Pingping.Formation Mechanism and Management Method of Workers' Overwork---on the Basis of Work Requiremen-the Analysis of a Resource Model[J].2010(9):30-31. 